University of Wollongong

Research Online

Faculty of Engineering - Papers (Archive)

Faculty of Engineering and Information

Sciences

$31-8-2001$

\title{
Magneto-optical Franz-Keldysh effect of an electron gas subjected to quantizing magnetic fields and intense terahertz laser fields
}

W. Xu

University of Wollongong

Follow this and additional works at: https://ro.uow.edu.au/engpapers

Part of the Engineering Commons

https://ro.uow.edu.au/engpapers/240

\section{Recommended Citation}

Xu, W.: Magneto-optical Franz-Keldysh effect of an electron gas subjected to quantizing magnetic fields and intense terahertz laser fields 2001.

https://ro.uow.edu.au/engpapers/240

Research Online is the open access institutional repository for the University of Wollongong. For further information contact the UOW Library: research-pubs@uow.edu.au 


\title{
Magneto-optical Franz-Keldysh effect of an electron gas subjected to quantizing magnetic fields and intense terahertz laser fields
}

\author{
W. $\mathrm{Xu}^{*}$ \\ Department of Engineering Physics, University of Wollongong, Wollongong NSW 2522, Australia \\ (Received 22 February 2001; published 31 August 2001)
}

\begin{abstract}
When a three-dimensional electron gas is subjected to quantizing magnetic fields and intense laser fields in the Faraday geometry, the maximum electron density of states will be shifted by an energy $E_{\text {em }}$ $=\left(e F_{0}\right)^{2} /\left[4 m^{*}\left(\omega^{2}-\omega_{c}^{2}\right)\right]$, where $\omega$ and $F_{0}$ are, respectively, the frequency and the electric-field strength of the laser field and $\omega_{c}$ is the cyclotron frequency. This will result in a shift in the fundamental absorption edge by $E_{\mathrm{em}}$, known as the magneto-optical Franz-Keldysh effect (MOFKE). In this paper, the MOFKE is studied theoretically for a semiconductor-based electron gas system where terahertz free-electron lasers are employed as intense laser radiation sources.
\end{abstract}

DOI: 10.1103/PhysRevB.64.113310

PACS number(s): 78.20.Ls, 71.20.-b, 78.40.Fy

In recent years, there has been a rapid expansion in the development of coherent, high-power, long-wavelength, and frequency-tunable radiation sources such as terahertz $(\mathrm{THz})$ or far-infrared free-electron lasers (FEL's). The current generation of FEL's can provide linearly polarized laser radiation in the form of a pulsed and/or continuum wave. Since 1995, the THz FEL's have been successfully applied in scientific research into nonlinear transport and optical properties in different semiconductor devices. ${ }^{1}$ Motivated by the development and application of these state-of-the-art laser technologies, in this paper, I report on the theoretical study of how electrons in a semiconductor response to linearly polarized intense $\mathrm{THz}$ laser fields in the presence of strong static magnetic fields.

It is well known that when an electron gas is subjected to a strong d.c. field, the electron density of states (DOS) will be blueshifted by the energy of the electric field. As a consequence, the fundamental absorption edge will also be blueshifted, which is known as the Franz-Keldysh effect (FKE). ${ }^{2}$ A similar phenomena occurs when an intense laser field is applied to an electron gas system, where the electron DOS and the fundamental absorption edge are blueshifted ${ }^{3,4}$ by an energy $E_{\mathrm{em}}=\left(e F_{0}\right)^{2} /\left(4 m^{*} \omega^{2}\right)$, where $m^{*}$ is the effectiveelectron mass and $\omega$ and $F_{0}$ are, respectively, the frequency and the electric-field strength of the radiation field. This is electrically analogous to the FKE and the effect has been named as the dynamical Franz-Keldysh effect (DFKE) ${ }^{5}$ Very recently, the blueshift of the fundamental absorption edge by $E_{\text {em }}$ in GaAs-based two-dimensional electron gas (2DEG) systems has been successfully observed experimentally using THz FEL's as intense laser radiation sources. ${ }^{6}$

A recently achieved experimental setup has made it possible to perform transport and optical measurements of a semiconductor device in strong magnetic fields and THz FEL fields. ${ }^{7}$ When we consider that an electronic system is subjected simultaneously to quantizing magnetic fields and to intense $\mathrm{THz}$ laser fields, we enter a regime with different competing energies, such as the Fermi, cyclotron, photon, plasmon, and phonon energies. These energies (frequencies) can be of the order of $\mathrm{meV}(\mathrm{THz})$. This offers us the possibility of observing $\mathrm{THz}$ photon-induced novel quantum effects. In fact, the distinctive features of an electronic system in high magnetic fields and intense laser fields have been noticed in early theoretical work ${ }^{8}$ where the electronic structure and impurity states were investigated. In this paper, I examine a rather simple phenomena that may be observable using the state-of-the-art laser and high magnetic-field technologies.

As in other studies, in the investigation of electronic and optical properties of an electron gas in strong magnetic fields and intense laser fields, the electron DOS is one of the central quantities required to determine and to understand almost all physically measurable properties. In the present paper, I employ a nonperturbative approach to obtain the electron DOS for an ideal three-dimensional electron gas (3DEG) in the presence of strong magnetic fields and intense laser fields in the Faraday geometry. I consider the situation where a magnetic-field $B$ and a laser field $A_{x}(t)$ are applied along the $z$ axis of a 3DEG, and the laser field is polarized linearly along the $x$ direction. In this configuration (known as the Faraday geometry), the magnetic field couples to the laser field and, as a result, cyclotron resonance (CR) effect can be expected. In this case, the most convenient gauge for describing the two uniform fields is

$$
\phi(\mathbf{R}, t)=0 \text { and } \mathbf{A}(\mathbf{R}, t)=\left[A_{x}(t), B x, 0\right],
$$

where $\mathbf{R}=(x, y, z)$. After using the dipole approximation for the electromagnetic (EM) field, we can write $A_{x}(t)$ $=A_{0} \sin (\omega t)$, where $\omega$ is the frequency of the radiation and $A_{0}=F_{0} / \omega$ with $F_{0}$ being the electric-field strength of the EM field. Thus, the electron Hamiltonian can be written as

$$
H(t)=\frac{1}{2 m^{*}}\left[\left\{p_{x}-e A_{x}(t)\right\}^{2}+\left(p_{y}-e B x\right)^{2}+p_{z}^{2}\right],
$$

where $m^{*}$ is the effective electron mass and $p_{x}=-i \hbar \partial / \partial x$ is the momentum operator. The time-dependent Schrödinger equation

$$
i \hbar \partial \Psi(\mathbf{R}, t) / \partial t=H(t) \Psi(\mathbf{R}, t)
$$

can be solved analytically and the time-dependent electron wave function is obtained as 


$$
\begin{aligned}
\Psi_{N, k_{y}, k_{z}}(\mathbf{R}, t)= & e^{i\left(k_{y} y+k_{z} z\right)} e^{-i E_{\mathrm{em}} \tau_{0}(t) / \hbar} e^{-i\left(E_{n}+E_{k_{z}}+E_{\mathrm{em}}\right) t / \hbar} \\
& \times \chi_{N}(x-X) e^{i x_{0}(x-X) / l^{2}}
\end{aligned}
$$

Here, $k_{y}$ is the electron wave vector along the $y$ direction, $E_{k_{z}}=\hbar^{2} k_{z}^{2} / 2 m^{*}, \quad X=X(t)=-l^{2} k_{y}+x_{1}(t) \quad$ with $l$ $=(\hbar / e B)^{1 / 2}$ being the radius of the ground cyclotron orbit, and $E_{N}=(N+1 / 2) \hbar \omega_{c}$ is the Landau level (LL) energy with $N=0,1,2, \ldots$, and $\omega_{c}=e B / m^{*}$ being the cyclotron frequency. Furthermore, in Eq. (3)

$$
\begin{gathered}
x_{0}=x_{0}(t)=-\frac{e F_{0}}{m^{*} \omega} \frac{\omega_{c} \sin (\omega t)-\omega \sin \left(\omega_{0} t\right)}{\omega^{2}-\omega_{c}^{2}}, \\
x_{1}=x_{1}(t)=\frac{e F_{0}}{m^{*}} \frac{\cos (\omega t)-\cos \left(\omega_{c} t\right)}{\omega^{2}-\omega_{c}^{2}}, \\
\tau_{0}(t)=\frac{\omega_{c}^{2} / \omega}{\omega^{2}-\omega_{c}^{2}}\left[\frac{3 \omega_{c}^{2}-\omega^{2}}{2 \omega_{c}^{2}} \sin (2 \omega t)+\frac{\omega}{\omega_{c}} \sin \left(2 \omega_{c} t\right)\right. \\
\left.-4 \sin (\omega t) \cos \left(\omega_{c} t\right)\right] \\
E_{\mathrm{em}}=\frac{\left(e F_{0}\right)^{2}}{4 m^{*}\left(\omega^{2}-\omega_{c}^{2}\right)}
\end{gathered}
$$

is an energy induced by the radiation and magnetic fields, and

$$
\chi_{N}(x)=\left(2^{N} N ! \pi^{/ 2} l\right)^{-1 / 2} e^{-(x / l)^{2} / 2} H_{N}(x / l),
$$

with $H_{N}(x)$ being the Hermite polynomials.

With the time-dependent electron wave function, we can derive the retarded propagator or Green's function for electrons in $(\mathbf{R}, t)$ - or (space, time)-representation

$$
\begin{aligned}
G\left(\mathbf{R}, t ; \mathbf{R}^{\prime}, t^{\prime}\right)= & -\frac{i}{\hbar} \Theta\left(t-t^{\prime}\right) \sum_{N, k_{y}, k_{z}} \\
& \Psi_{N, k_{y}, k_{z}}^{*}\left(\mathbf{R}^{\prime}, t^{\prime}\right) \Psi_{N, k_{y}, k_{z}}(\mathbf{R}, t),
\end{aligned}
$$

which satisfies

$$
\left[i \hbar \frac{\partial}{\partial t}-H(t)\right] G\left(\mathbf{R}, t ; \mathbf{R}^{\prime}, t^{\prime}\right)=\delta\left(t-t^{\prime}\right) \delta\left(\mathbf{R}-\mathbf{R}^{\prime}\right) .
$$

The Green's function in $(\mathbf{K}, t)$ - or (momentum, time)representation, $G\left(\mathbf{K} ; t, t^{\prime}\right)$ with $\mathbf{K}=\left(k_{x}, k_{y}, k_{z}\right)$, can be obtained by the space Fourier transform of $G\left(\mathbf{R}, t ; \mathbf{R}^{\prime}, t^{\prime}\right)$. After averaging $G\left(\mathbf{K} ; t, t^{\prime}\right)$ over $\mathbf{K}$, we obtain the Green's function in time representation

$$
\begin{aligned}
G\left(t, t^{\prime}\right)= & G(\tau, T) \\
= & -\frac{i}{\hbar} \frac{\Theta(\tau)}{2 \pi l^{2}} \sum_{N, k_{z}} e^{-i\left(E_{N}+E_{k_{z}}+E_{\mathrm{em}}\right) \tau / \hbar} \\
& \times e^{-i \mathcal{A}} e^{-\mathcal{B} / 2} L_{N}(\mathcal{B}),
\end{aligned}
$$

where $\tau=t-t$ ' is the time "relative coordinates," $T=(t$ $\left.+t^{\prime}\right) / 2$ is the time "center of mass," $L_{N}(x)$ is the Laguerre Polynomial,

$$
\mathcal{A}=\frac{E_{\mathrm{em}}}{\hbar \omega}\left[\frac{2 \omega_{c}^{2}}{\omega^{2}-\omega_{c}^{2}}-\cos (2 \omega T)\right],
$$

and

$$
\mathcal{B}=\frac{4 E_{\mathrm{em}} \omega_{c}}{\hbar \omega^{2}}\left[\frac{\omega^{2}+\omega_{c}^{2}}{\omega^{2}-\omega_{c}^{2}}-\cos (2 \omega T)\right] \sin ^{2}\left(\frac{\omega \tau}{2}\right) .
$$

The Fourier transform (or average over time $\tau$ ) of $G(\tau, T)$ is given by

$$
G(E, T)=\frac{1}{2 \pi l^{2}} \sum_{N, k_{z}} \sum_{m=-\infty}^{\infty} \frac{F_{N m}[\cos (2 \omega T)]}{E-E_{N}-E_{k_{z}}-E_{\mathrm{ern}}-m \hbar \omega+i \delta}
$$

where an infinitesimal quantity $i \delta$ has been introduced to make the integral converge, $E$ is the electron energy and

$$
\begin{aligned}
F_{N m}(y)= & \frac{(-1)^{m}}{\pi} \int_{0}^{\pi} d x \cos (m x+\mathcal{C} \sin x) \\
& \times e^{-\mathcal{D}(1+\cos x) / 4} L_{N}\left[\frac{\mathcal{D}}{2}(1+\cos x)\right],
\end{aligned}
$$

with

$$
\mathcal{C}=\frac{E_{\mathrm{em}}}{\hbar \omega}\left[\frac{2 \omega_{c}^{2}}{\omega^{2}-\omega_{c}^{2}}-y\right] \text { and } \mathcal{D}=\frac{4 E_{\mathrm{em}} \omega_{e}}{\hbar \omega^{2}}\left[\frac{\omega^{2}+\omega_{c}^{2}}{\omega^{2}-\omega_{c}^{2}}-y\right] .
$$

In Eq. (7), $m$ is induced by the Fourier transform and corresponds to emission and absorption of photons with energy $m \hbar \omega$. Therefore, $m$ is an index for different optical channels, i.e., $m>0(m<0)$ for $m$-photon absorption (emission) and $m=0$ for elastic optical process. By averaging $T$ in Eq. (7) over a period of the radiation field, the Green's function in spectrum representation is obtained as

$$
G(E)=\frac{1}{2 \pi l^{2}} \sum_{N, k_{z}} \sum_{m=-\infty}^{\infty} \frac{F_{N m}}{E-E_{N}-E_{k_{z}}-E_{\mathrm{em}}-m \hbar \omega+i \delta},
$$

where $F_{N m}=(1 / 2 \pi) \int_{0}^{2 \pi} d x F_{N m}[\cos (2 x)]$.

The steady-state electron DOS is determined by the imaginary part of the retarded Green's function in spectrum representation, which reads

$$
\begin{aligned}
D(E)= & \frac{\left(2 m^{*} / \hbar^{2}\right)^{1 / 2}}{2 \pi^{2} l^{2}} \sum_{N=0}^{\infty} \sum_{m=-\infty}^{\infty} F_{N m} \\
& \times \frac{\Theta\left(E-E_{N}-E_{\mathrm{em}}-m \hbar \omega\right)}{\sqrt{E-E_{N}-E_{\mathrm{em}}-m \hbar \omega}} .
\end{aligned}
$$


For the case of a high-frequency $(\omega \gg 1)$ and/or a lowintensity $\left(F_{0} \ll 1\right)$ radiation field, entailing $E_{\mathrm{em}} \rightarrow 0$, Eq. (9) becomes that obtained in the absence of the radiation field.

In the nonperturbative approach developed here in obtaining the Green's function for electrons and the electron DOS, the effects of the magnetic and radiation fields are included exactly. In an electronic system, when magnetic field couples to the radiation field, the CR effect should be present. This can be seen in the time-dependent electron wave function [Eq. (3)], the Green's function for electrons [Eqs. (4), (6)(8)] and in the electron DOS [Eq. (9)]. In the presence of the radiation fields, electrons in the system can interact with the radiation field via absorption and emission of photons, including multiphoton processes. This has been reflected in the Green's function [Eq. (8)] and in the electron DOS [Eq. (9)], where a factor $F_{N m}$ plays a role in switching different optical channels for different LL's. Furthermore, the coupling of the magnetic and radiation fields to the electronic system results in the energy of the system being shifted by the energy $E_{\mathrm{em}}$. This implies that for a 3DEG subjected to a strong magnetic field and an intense laser field, the maximum electron DOS will be shifted by the energy $E_{\text {em }}$ and, as a result, the fundamental absorption edge will also be shifted by $E_{\mathrm{em}}$. Thus, for the present situation, the Franz-Keldysh effect should be observed through the shift of the fundamental absorption edge by an energy $E_{\mathrm{em}}=\left(e F_{0}\right)^{2} /\left[4 m^{*}\left(\omega^{2}-\omega_{c}^{2}\right)\right]$. Since this effect occurs in the presence of coupled magnetic and laser fields and is fundamentally different from those observed in the absence of the magnetic field, we call it here as magnetooptical Franz-Keldysh effect (MOFKE).

In contrast to the DFKE observed in the absence of a magnetic field, where only a blueshift can be observed, the presence of the magnetic field will lead to a phenomenon that both blue $\left(\omega>\omega_{c}\right)$ and red $\left(\omega<\omega_{c}\right)$ shifts can be measured in the MOFKE. When the condition of the CR is sat-

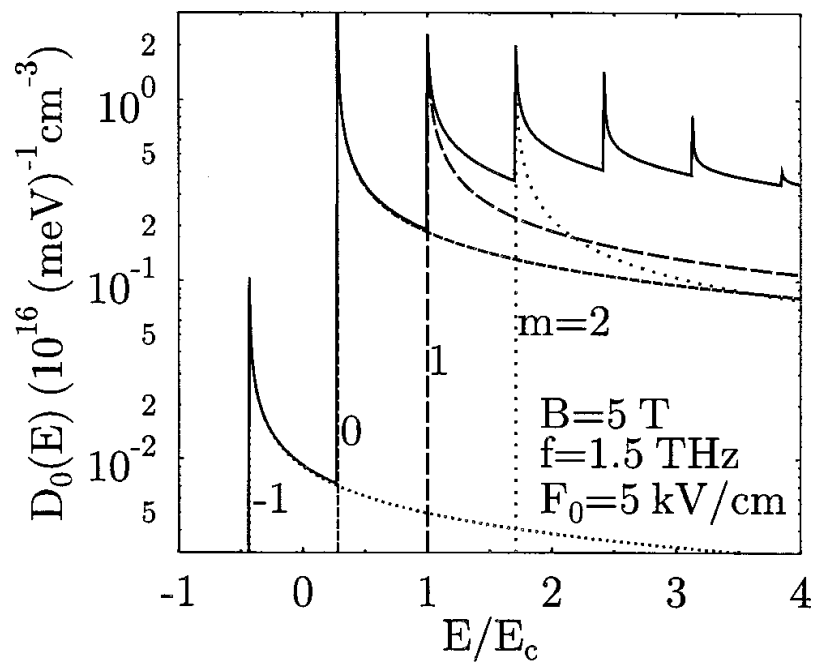

FIG. 1. Contribution from different optical channels to electron density of states (DOS) for the Landau level (LL) $N=0$ at a fixed magnetic field and a fixed radiation field. The solid curve is the total DOS for the 0th LL, $m>0(m<0)$ corresponds to a channel for $m$-photon absorption (emission), and $E_{c}=\hbar \omega_{c}$.

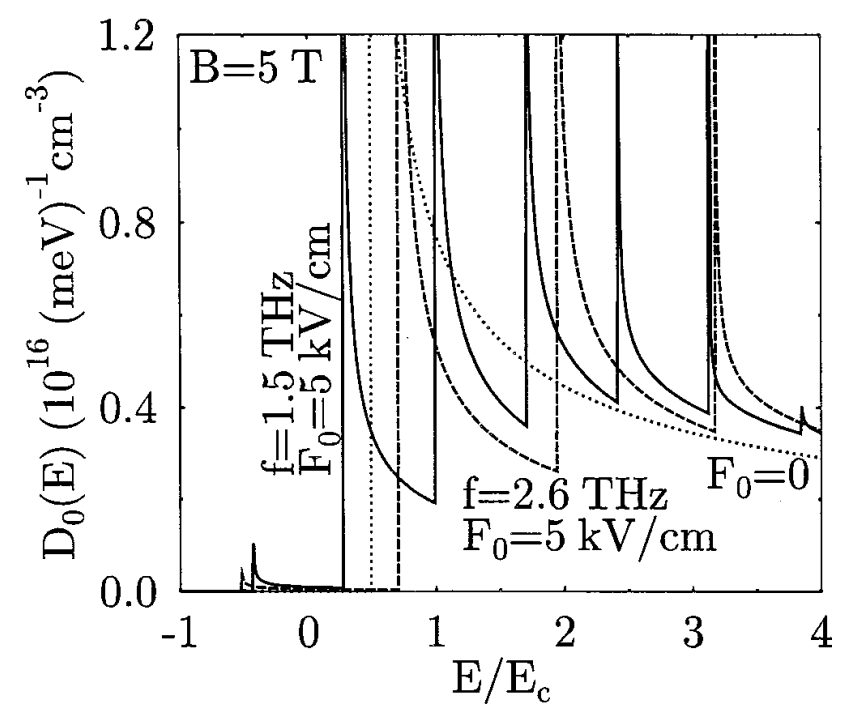

FIG. 2. Electron DOS for the 0th LL at a fixed magnetic field for different radiation fields: (1) $f=1.5 \mathrm{THz}$ and $F_{0}=5 \mathrm{kV} / \mathrm{cm}$ (solid curve); (2) $f=2.6 \mathrm{THz}$ and $F_{0}=5 \mathrm{kV} / \mathrm{cm}$ (dashed curve); and (3) $F_{0}=0$ (dotted curve) corresponds to the case in the absence of the radiation field and the maximum DOS appears at $E=E_{c} / 2 . E_{c}$ $=\hbar \omega_{c}$.

isfied, i.e., $\omega \sim \omega_{c}$, the electronic transitions are mainly achieved via inter-LL transition events and the shift of the MOFKE cannot be measured.

Now I will discuss numerical results obtained for a GaAsbased 3DEG system. In Fig. 1, the contribution from different optical channels to the electron DOS is shown for the lowest LL $N=0$ at a fixed magnetic field and a fixed radiation field. It should be noted that (1) the maximum electron

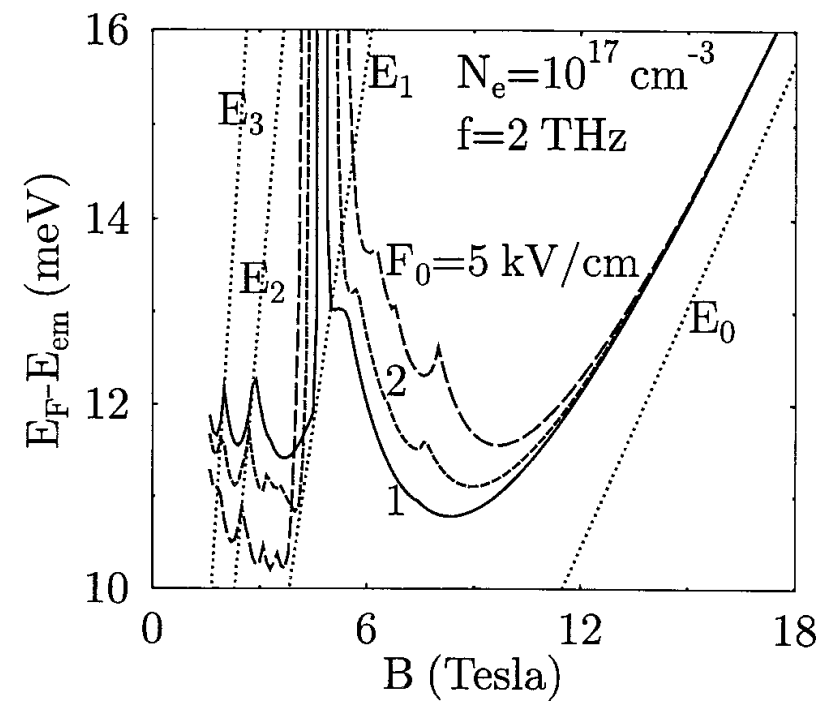

FIG. 3. Fermi energy, measured from the energy $E_{\text {em }}$, versus magnetic field at a field radiation frequency for different radiation intensities. $N_{e}$ is the electron density and $E_{N}$ is the $N$ th LL energy. Note that the cyclotron resonance effect occurs at $\omega_{c}=\omega$. 
DOS appears when a channel for elastic optical scattering (i.e., $m=0$ ) opens up; (2) in the presence of the radiation field, the electron DOS can be present in the lower and even negative energy regime, due to the presence of channels for optical emission; and (3) a step increase in the DOS can be observed when an optical channel for $m$-photon absorption or emission opens up. In Fig. 2, the electron DOS for the 0th LL is shown at a fixed magnetic field for different radiation fields. $F_{0}=0$ (dotted curve) corresponds to the case in the absence of the radiation field. We see that at a fixed radiation intensity $F_{0}$, red (solid curve) and blue (dashed curve) shifts of the DOS can be achieved via varying the radiation frequency. We can determine the Fermi energy of the system by introducing the electron DOS into the condition of electron number conservation. The Fermi energy, measured from the energy $E_{\mathrm{em}}$, is plotted in Fig. 3 as a function of magnetic field at a fixed radiation frequency for different radiation intensities. Here it is noted that (i) with increasing radiation intensity, a stronger CR effect is observed in Fermi level; (ii) when $\omega_{c}>\omega\left(\omega_{c}<\omega\right)$ the Fermi energy increases (decreases) with increasing $F_{0}$; and (iii) some fine structures induced by different optical processes can also be observed in the Fermi energy.

In this paper, I have demonstrated that when an electron gas is subjected to intense laser fields and to strong magnetic fields, the maximum electron DOS and, as a result, the fundamental absorption edge will be shifted by an energy $E_{\text {em }}$ $=\left(e F_{0}\right)^{2} /\left[4 m^{*}\left(\omega^{2}-\omega_{c}^{2}\right)\right]$. In contrast to the DFKE observed at $B=0$ where only a blueshift can be seen, I predict that in the presence of the strong magnetic fields, both red and blue shifts of the MOFKE can be measured. For a semiconductor system, the MOFKE can be observed when $B$ $\sim 1 \mathrm{~T}, F_{0} \sim 10 \mathrm{kV} / \mathrm{cm}$ and $\omega \sim 1 \mathrm{THz}$. The radiation condition has been realized by the current generation of the $\mathrm{THz}$ FEL's. I therefore hope that the phenomena predicted in this paper will be verified experimentally.

The author is a Research Fellow of the Australian Research Council (ARC). This work was also supported by the ARC Large Grant and IREX Grant. Discussions with X. L. Lei (SJTU) are gratefully acknowledged.
*Electronic mail: wxu@wumpus.its.uow.edu.au

${ }^{1}$ See, e.g., C. J. G. M. Langerak, B. N. Murdin, B. E. Cole, J. M. Chamberlain, M. Henini, M. Pate, and G. Hill, Appl. Phys. Lett. 67, 3453 (1995); N. G. Asmar, A. G. Markelz, E. G. Gwinn, J. Gerne, M. S. Sherwin, K. L. Campman, P. E. Hopkins, and A. C. Gossard, Phys. Rev. B 51, 18041 (1995).

${ }^{2}$ See, e.g. K. Seeger, Semiconductor Physics-An Introduction (Springer-Verlag, Berlin, 1991).

${ }^{3}$ For the case of a 3DEG, W. Xu, Phys. Rev. B 57, 15282 (1998).

${ }^{4}$ For the case of a 2DEG, W. Xu, Semicond. Sci. Technol. 12, 1559 (1997).
${ }^{5}$ A. P. Jauho and K. Johnsen, Phys. Rev. Lett. 76, 4576 (1996).

${ }^{6}$ K. B. Nordstrom, K. Johnsen, S. J. Allen, A. P. Jauho, B. Birnir, J. Kono, T. Noda, H. Akiyama, and H. Sakaki, Phys. Rev. Lett. 81, 457 (1998).

${ }^{7}$ P. M. Koenraad, R. A. Lewis, L. R. C. Waumans, C. J. G. M. Langerak, W. Xu, and J. H. Wolter, Physica B 256-258, 268 (1998).

${ }^{8}$ P. Hawrylak and L. Rego, Physica E (Amsterdam) 3, 198 (1998); P. Hawrylak, P. A. Schulz, and J. J. Palacios, Solid State Commun. 93, 909 (1995). 